\title{
Refined genotype-phenotype correlations in cases of chromosome 6p deletion syndromes
}

\author{
Ghazala Mirza $^{1}$, Ruth R. Williams ${ }^{2}$, Shela Mohammed ${ }^{2}$, Robin Clark ${ }^{3}$, \\ Ruth Newbury-Ecob ${ }^{4}$, Shari Baldinger ${ }^{5}$, Frances Flinter ${ }^{2}$ and Jiannis Ragoussis ${ }^{\star, 1}$
}

\begin{abstract}
${ }^{1}$ Wellcome Trust Centre for Human Genetics, University of Oxford, Department of Genomics, Roosevelt Drive, Headington, Oxford OX3 7BN, UK; ${ }^{2}$ Division of Medical \& Molecular Genetics, Guy's Hospital Tower, Guy's, King's Hospital and St. Thomas' School of Medicine, London SE1 9RT, UK; ${ }^{3}$ USC/Keck School of Medicine, Cancer Genetics Units, USC/Norris Comprehensive Cancer Center, Los Angeles, CA 90089, USA; ${ }^{4}$ Genetics Centre, Royal United Hospital, Combe Park, Bath BA1 3NG, UK; ${ }^{5}$ Abbott-NW Hospital, 800E, 28 ${ }^{\text {th }}$ St, IMPLS, MN 54507, USA
\end{abstract}

Clinical reports of cases with deletions in chromosome $6 p$ are relatively rare. We present a detailed study by fluorescent in situ hybridisation (FISH) of six new cases with distinct but overlapping 6p deletions involving the 6p24-pter chromosomal segment. Chromosomal breakpoints in individual cases were investigated using a large panel of probes previously mapped and characterised in our laboratory to cover the distal region of $6 \mathrm{p}$. These cases have allowed refinement of genotype-phenotype correlations and strongly suggest a gene involved in regulating the development of hearing is localised within $6 \mathrm{p} 25$. There is also evidence for one or more loci involved in heart, skeletal and craniofacial development in the 6p24p25 region. Furthermore, the Dandy-Walker malformation is associated with deletion of 6p24-pter. European Journal of Human Genetics (2004) 12, 718-728. doi:10.1038/sj.ejhg.5201194

Published online 19 May 2004

Keywords: $6 p$ deletion syndromes; sensorineural deafness; mouse models

\section{Introduction}

Chromosome $6 \mathrm{p}$ deletions are rare events within the population. At present, there are 43 cases in the medical literature, excluding ring chromosome 6 anomalies. These include 27 reports on terminal deletions of $6 p$, six cases on unbalanced translocations, and the remaining 10 cases describe interstitial deletions with the entire 6p22, $6 \mathrm{p} 22.2-\mathrm{p} 25.2$ or $6 \mathrm{p} 24-\mathrm{p} 25$ segment deleted ${ }^{1-11}$ (see references therein).

The clinical findings vary in the interstitial deletion $6 \mathrm{p}$ syndromes, and include orofacial clefting, short neck, clinodactyly or syndactyly and brain, heart and kidney defects. In contrast, corneal opacity, iridogoniodysgenesis

*Correspondence: J Ragoussis, Wellcome Trust Centre For Human Genetics, Department of Genomics, University of Oxford, Roosevelt Drive, Headington, UK. Tel: +44 1856 287526; Fax: + 441856 287533; E-mail: ioannis.ragoussis@well.ox.ac.uk

Received 17 June 2003; revised 18 February 2004; accepted 24 February 2004 anomaly, various Rieger type anomalies, hypertelorism and deafness are associated with $6 \mathrm{p}$ terminal deletions.

A large number of mouse model studies have provided valuable insights into the function of genes located in the syntenic segment of mouse chromosome 13, including roles in organogenesis, and in particular eye, cardiac and skeletal development. ${ }^{12-17}$

In this paper, we describe six new cases that enable us to delineate the $6 \mathrm{p}$ deletion syndrome involving the $10 \mathrm{Mbp}$ long 6p24-pter chromosomal segment with further accuracy. Our findings allow us to associate developmental defects (see listing in Discussion) to deletions of specific chromosomal segments and to candidate genes therein.

\section{Patient 1}

The youngest of two siblings (DOB 08/11/95) was born after an uneventful pregnancy and delivery: the patient was male, with a birth weight of $3.6 \mathrm{~kg}$ (50th centile), head 
circumference was $49.8 \mathrm{~cm}$ (25th-50th centiles) and height was on the 25 th centile.

\section{Symptoms at birth}

Hypertelorism and down-slanting palpebral fissures.

\section{Initial cytogenetic data}

G-banded preparations showed that the subtelomeric segment was absent from the derivative chromosome 6 . Subsequent in situ hybridisation confirmed the initial karyotype: 46,XY,del(6)(p25.1). This event is de novo as both parents' karyotypes were normal.

\section{Additional clinical data}

Ophthalmolgical assessment revealed strabismus (which recurred after surgery) and marked posterior embryotoxon of both eyes. Anterior fibres from the iris adhered to the embryotoxon in places, but the anterior chamber was otherwise normally formed. The intra ocular pressure was normal.

The patient's ears were slightly low set and posteriorly rotated (Figure 1). A CT scan of the ears showed a poorly pneumatised mastoid on the left side. He had a broad nasal bridge, a highly arched palate, abnormal dentition.

Physical examination at 5 years noted a harsh systolic and a very soft early diastolic murmur after the aortic component of the second sound. Echocardiography demonstrated a minor structural abnormality of his aortic valve, though it was functioning normally.

He also had a left-sided cervical skin tag, slightly broad toes and his audiometry revealed bilateral mild to moderate low-frequency sensorineural hearing loss. He had moderate learning difficulties, language delay and motor milestones were delayed, as he sat at 11 months and walked at around approximately 16 months. A Griffith's assessment (14-01-99) showed him to be a year behind his chronological age of 4 years (see Table 1 for summary of phenotypes).

\section{Patient 2}

Female patient (DOB 3/05/00) was born to a 29-year-old woman after a full-term delivery, complicated by abruptio a

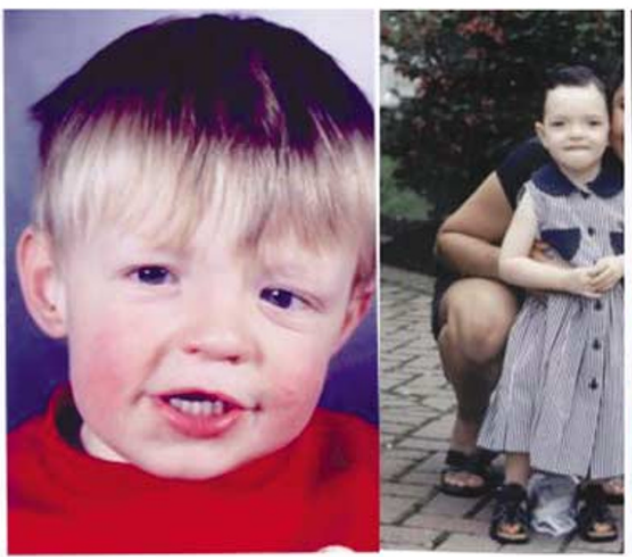

d

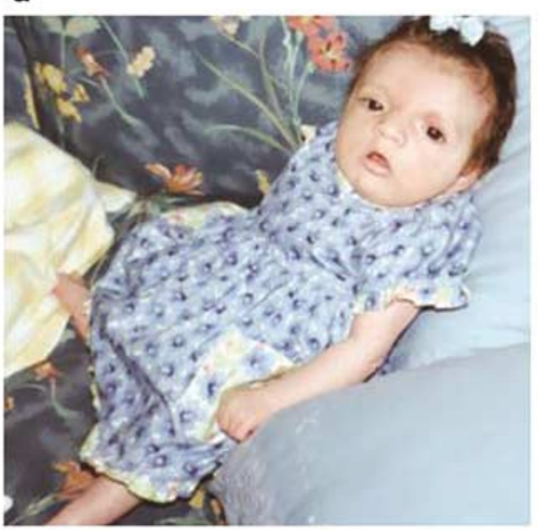

C

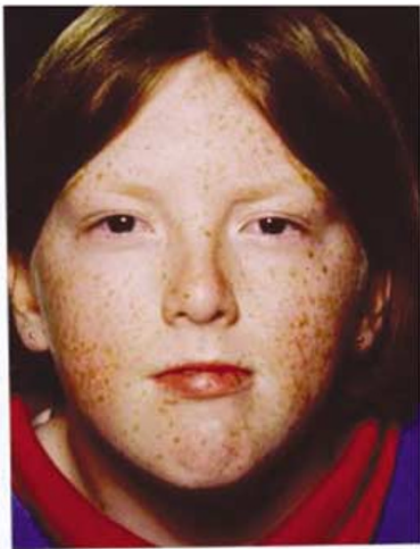

e

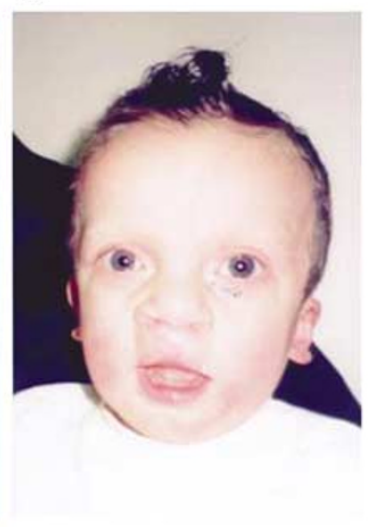

Figure 1 Photographs of five of the $6 p$ deletion cases; (a) Patient 1; (b) Patient 2; (c) Patient 3; (d) Patient 5; (e) Patient 6. 
Table 1 Main characteristics of chromosome $6 p$ deletion cases presented in this study

\begin{tabular}{|c|c|c|c|c|c|c|}
\hline Patients Clinical Features & 1 & 2 & 3 & 4 & 5 & 6 \\
\hline Sex & Male & Female & Female & Female & Female & Male \\
\hline $\begin{array}{l}\text { Abnormal skull } \\
\text { shape/hydrocephalus }\end{array}$ & & & & + & & \\
\hline shape/hydrocephalus & & & + & & & \\
\hline Hypertelorism & + & + & + & & + & + \\
\hline Palpebral fissures defects & + & & + & & & \\
\hline Palatal abnormalities & + & & & & + & + \\
\hline Dental abnormalites & + & + & + & & & \\
\hline Structural ear anomalies & + & & & & & + \\
\hline Structural nose defects & + & & & & & + \\
\hline Skin anomalies & & & & & & + \\
\hline Skin tags & + & & & & & \\
\hline Heart defect & + & + & & + & + & + \\
\hline Imperforate anus & & & & & & + \\
\hline Neuronal defect & + & + & + & + & & \\
\hline Motor delay & + & + & + & & + & \\
\hline Extremities anomalies & + & & + & & & + \\
\hline Language delay & + & + & & & & \\
\hline
\end{tabular}

placenta requiring emergency caesarean. Birth weight was $3.2 \mathrm{~kg}$ (45th centile).

\section{Symptoms at birth}

Hypotonia, facial dysmorphism, abnormal creases in the hand and feet and hyperflexion of both feet.

\section{Initial cytogenetic data}

Cytogenetic analysis using G-banded chromosomes revealed the karyotype: $46, \mathrm{XX}$, $\operatorname{del}(6)(\mathrm{p} 24)$. This event is de novo as both parents' karyotypes were normal.

\section{Additional clinical data}

At 20 months, she was treated for pneumonia and had been diagnosed with asthma.

Examination at 2 years revealed a prominence in the occipital area, hypertelorism, hypermetropia and a strabismus (Figure 1). The teeth were abnormal and low in enamel. She had a carped shaped mouth, a small patent ductus arteriosus (PDA) and patent foramen ovale (PFO). Both feet had a rocker bottom appearance with bilateral ankle pronation. Hypotonia and joint hyperlaxity conditions were still present.

Gross motor skills were judged to be at 15 months level as she was not able to walk unassisted at 2 years. Adaptive skills were judged to be at 16-19 months level, cognitive skills were normal at 2 years. Skeletal development was showing delay and the anterior fontanel had not closed. She displayed hypersensitivity to temperature, auditory hypersensitivity and the mouth showed poor response to stimulation. A CT scan revealed a prominence in the fourth ventricle with questionable changes in the cerebella vermis and cerebella white matter.

In contrast to the parents, who are Hispanic, the baby was very fair. Thyroid stimulating hormone (TSH) levels were high, a blood test indicated high immunoglobulin $\mathrm{E}$ (IgE) levels and diagnosed anaemia. She was undergoing occupational and speech therapy in addition to physiotherapy.

Her proprioceptor input showed hyperactive irregularity and she displayed seizures that occur with a frequency of around 60 per day lasting for up to $1 \mathrm{~min}$ per seizure. It is thought these seizures were non specific and can occur in children with a developmental delay (see Table 1 for summary of phenotypes).

\section{Patient 3}

A girl, the eldest of two siblings (DOB 13-01-84), was born after an uneventful pregnancy and delivery, with a birth weight of $4.0 \mathrm{~kg}$ ( $>90 \mathrm{th}$ centile). The parents are non consanguineous and show no apparent family history of malformations. She presented at the age of 1 year with developmental delay and concerns about her hearing and vision.

\section{Initial cytogenetic data}

At 5 years, her chromosomes were analysed and a de novo del (6) p25 $\rightarrow$ pter karyotype was identified. 


\section{Additional clinical data}

Examination at 13 years revealed macrocephaly (head circumference $56.5 \mathrm{~cm}$ ), upslanting short palpebral fissures, prominent epicanthic folds, mild dysmorphic features and a short philtrum. Eye examination revealed telecanthus, anterior segment dysgenesis, a divergent squint, hypermetropia, and mild pigmentary retinopathy (Figure 1). The teeth were prominent and overcrowded. Her toes were clawed, with a short second toe and bilateral fifth finger clinodactyly. She also had hip dysplasia, diagnosed as bilateral Perthes disease. She had undergone tonsillectomy, adenoidectomy, insertion of grommets and had previously suffered from faecal soiling secondary to severe constipation and overflow diarrhoea. In addition, she suffered from conductive hearing loss, with impaired motor skills and slow speech.

\section{Patient 4}

A second child, female was born to healthy nonconsanguineous parents (DOB 14-12-00), with a birth weight of 3 . $7 \mathrm{~kg}$ (60th centile).

\section{Symptoms at birth}

Examination at birth showed her to have hydrocephalus. She had been diagnosed with Dandy-Walker Syndrome with possible agenesis of the corpus callosum. A ventriculoperitoneal shunt had been inserted at 6 weeks of age.

\section{Initial cytogenetic data}

G-banded chromosome analysis revealed her to be: $46, \mathrm{XX}$, del $(6)($ p24.3 $\rightarrow$ pter $)$ : in situ hybridisation also confirmed this karyotype. The parents' metaphase chromosomes were also analysed, both were normal for the respective region, indicating a de novo event.

\section{Additional clinical data}

Corneal opacities consistent with Peter's anomaly: she received bilateral cornea transplants at 2 weeks of age. A ventricular septal defect (VSD) was diagnosed and subsequently repaired at age 4 weeks. A gastrointestinal tube was inserted for feeding at approximately 6 weeks of age (see Table 1 for summary of phenotypes).

Mild sensorineural hearing loss in the right ear (35 db) and moderate to severe hearing loss in the left ear $(60 \mathrm{db})$. Assessment at around 2 months indicated neurodevelopmental delay.

\section{Patient 5}

A female (DOB 02/04/02) was born to a 33- and 36-year-old mother and father, respectively. This was their first child and there was no clinical history of genetic abnormalities. Patient 5 was born at full term via a Caesarean section due to a breech presentation, with a birth weight of $3.54 \mathrm{~kg}$ (50th centile).

\section{Symptoms at birth}

Features present at birth included hypertelorism, a short neck, and a severely displaced hip: the latter condition possibly attributable to the breech presentation. Cerebral magnetic resonance imaging (MRI) revealed no significant abnormalities. Eye examinations, hearing tests and sonograms revealed no other abnormalities. The palate was highly arched. MRI detected some communication in the anterior flax. This was felt to have no clinical significance.

\section{Initial cytogenetic data}

Cytogenetic examination using G-banded chromosomes revealed a 46,XX,del(6)(p24.3) karyotype.

\section{Additional clinical data}

By 1 month of age, she had extensive hemangiomas and telangiectasias on the right arm and right shoulder girdle. A check up at 13 weeks showed occipital flattening, and mildly low set ears, mild pectus excavatum and mild hypotonia were present; radiographic examination showed the hips had healed. Patient 5 had suffered from numerous episodes of bronchiolitis and ear infections. A check up at 5 months revealed blue sclerae, hypotonia and loose joints (Figure 1). There was moderate motor delay, but otherwise, her growth, social and visual skills were good.

Examination of Patient 5 at 6 months showed relative brachycephaly and plagiocephaly appearance with right occipital bones somewhat more prominent than left, the anterior fontanel had not closed. The ears are posteriorly rotated and the nose was broad based. The abdomen was mildly protuberant with small fingertip-sized defect in the abdominal wall and umbilical hernia. The fingers were long and with ulnar deviation, the thumbs were bilaterally low set. The shoulder was particularly hyperflexible. The deep tendon reflexes were normal and symmetric throughout (see Table 1 for summary of phenotypes).

\section{Patient 6}

A first-born male (DOB 13-3-00) to a nonconsanguineous Hispanic mother and father of 23 and 28 years, respectively. The boy was born at 35 weeks gestation following treatment for preterm labour from 29 weeks.

\section{Symptoms at birth}

At birth, he had asymmetric flattening of the occiput, bilateral cleft lip, PDA, atrial septal defect (ASD); mild pelviectasis was noted on renal ultrasound and he had an imperforate anus. A brain stem auditory evoked response was normal. The bilateral cleft lip and imperforate anus were repaired in the first year. 
Initial cytogenetic data

Cytogenetic analysis using G-banded chromosomes revealed a de novo interstitial deletion that was initially reported as: 46XY,del (6)(p23p24.3) de novo.ish 6pter (TelVysion probe $6 \mathrm{p} \times 2$ ).

\section{Additional clinical data}

At $7 \frac{1}{2}$ months, physical examination showed hypertelorism with prominent eyes, flat supraorbital ridges and grey-blue sclerae (Figure 1), small posteriorly rotated, mildly dysplastic ears with bilateral preauricular pits. The left ear was overfolded and the right ear lacked the superior ramus of the antihelix. He had frontal upsweep of hair, a broad nose with a bulbous tip, but the palate was intact. There was mild pectus excavatum, a small umbilical hernia and an imperforate anus. The penis was small and testes were in the scrotum. Mild eczema was present. The middle toes were overlapping and thumbs were often adducted. The digits were otherwise normal except for spoon-shaped toenails. Neurological examination was normal except for mild drooling. Growth and development were essentially normal. At 13 months, he was at the 90th percentile for height and the 25th percentile for weight. Head circumference was also normal at the 10-25th percentile. At 13 months, the patient was taking one or two steps independently and he could say at least four words. His developmental assessment was age appropriate (see Table 1 for summary of phenotypes).

Family history included heart defects present in maternal second-degree relatives.

\section{Methods}

Tissue culture and fluorescent in situ hybridisation (FISH) were performed as described previously. ${ }^{1}$ In total, 13 YAC, 44 PAC, two BAC clones and one cosmid, that had been previously FISH mapped to defined locations on $6 \mathrm{p}$ or contained identifiable genetic markers and genes, were used. The PAC clones were chosen from the Sanger Institute's FPC database (http://www.sanger.ac.uk/cgi-bin/ humace/fpcwebmap, accessed May 2003); clones were ordered according to fingerprinting and marker content results. Clones present in Ensembl are a selection of the fingerprinted clones, and it is therefore possible to estimate the position of the clones used in this study in the Ensembl database (http://www.ensembl.org/, accessed May 2003). The YAC clones were ordered according to reported marker content by cross-referencing in both databases and confirmed by our own FISH-based clone ordering experiments. Additional data were compiled from the Ensembl database (May 2003) to describe genes identified so far on distal $6 \mathrm{p}$ with respect to their markers, known function, distance from the telomere and chromosomal localisation (see Table 2). Breakpoint positions in the cases described above are shown in Table 2 and reflect our best estimates.

\section{Results}

Deletions were confirmed by FISH as terminal or interstitial using probes mapping to 6p23-pter (Table 3). A dense collection of probes was used around regions where breakpoints occurred (Table 3). Clones were denoted as positive when a hybridisation signal was detected on the derivative chromosome 6 [del (6)] and negative when no signal was present.

Patient 1 has the smallest deleted $6 \mathrm{p}$ segment. YAC $952 \mathrm{~h} 4$, which localises in $6 \mathrm{p} 25.3$, was positive on the respective del (6). However, PAC dJ223B1, which lies distal to the YAC, did not produce a hybridisation signal on del (6). The breakpoint lies between these probes: based on this result, we have refined the cytogenetic diagnosis and positioned the breakpoint in 6p25.2 (Table 3).

Patient 2 has the deletion breakpoint positioned in PAC DJ287K15, just distal of YAC 878B10, which produces a positive signal on del (6) and lies proximal in $6 \mathrm{p} 25$ (Table 3).

Patient 3 has the breakpoint positioned within the proximal segment of $6 \mathrm{p} 25$ with all probes distal to PAC dJ23O21 proving negative on del (6) (Table 3).

Patient 4 has the largest deleted segment with the breakpoint occurring within 6p24.3. PAC dJ436h5 did not hybridise on del (6), the probe lies just distal to BAC 648 N19, which gave a positive signal on del (6) (Table 3). We were able to confirm the original cytogenetic diagnosis as 46XX,del (6)(p24.3).

PAC dJ118B18, which contains the FOXC1 gene and lies in the subtelomeric region of $6 \mathrm{p}$, was used for FISH on chromosomes of patient 5 . The results showed this distal region was indeed not deleted on del (6). The most proximal probe that did not hybridise on del (6) was PAC dJ133h11, this probe lies in $6 \mathrm{p} 24.3$, immediately distal to dJ167k8, that gave a positive signal on del (6) (Table 3). Thus, we were able to confirm the cytogenetic finding as 46XX,del (6)(p24.3p25.3).

The distal segment of $6 \mathrm{p} 25$ in Patient 6 was not deleted: instead, we have shown that the deletion is interstitial with BAC B18 marking the distal breakpoint and PAC 524G21 the proximal breakpoint (Table 3 ). Thus, we were able to refine the initial karyotype finding to 46XY,del (6)(p24p25.2). The extent of the deletion is less than previously thought from conventional cytogenetic methods.

Examples of FISH results of all cases are represented in Figure 2. Based on the FISH results and mapping data available from the Ensemble and the Sanger Centre's physical mapping database, the total amount deleted from chromosome 6 is: over $3.2 \mathrm{Mb}$ from Patient $1,5.8 \mathrm{Mb}$ from Patient 2, 6 Mb from Patient 3, $9 \mathrm{Mb}$ from Patient $4,6.9 \mathrm{Mb}$ from Patient 5 and $7 \mathrm{Mb}$ from Patient 6. This corresponds to $0.09,0.18,0.17,0.23,0.17$ and $0.14 \%$ of the total human genome deleted, respectively. The phenotypes of the collective cases and corresponding photographs are shown in Table 1 and Figure 1, respectively. 
Table 2 Data compiled from the Ensembl database (http://www.ensembl.org/), and the Sanger Institute's FPC database (see Methods for description)

\begin{tabular}{|c|c|c|c|c|c|}
\hline & Genes & Marker/clone information & Additional information & $\begin{array}{l}\text { Distance from } 6 p \text { Telomere } \\
(\mathrm{Mb})\end{array}$ & Chromosome location $6 p$ \\
\hline 1 & NOVEL & AL353654 & Similar to Septin & 0.117 & 25.2 \\
\hline 2 & Q9NRW4 & & Protein kinase phosphatase & 0.277 & 25.2 \\
\hline 3 & IRF4 & & Interferon Regulatory Factor 4 & 0.377 & 25.2 \\
\hline 4 & Q96KP1 & & Ambiguous & 0.471 & 25.2 \\
\hline 5 & QUJM7 & & Exocyst Complex component & & 25.2 \\
\hline 6 & NÓVEL & AL031770 & Unknown & 0.587 & 25.2 \\
\hline 7 & Q9C009 & AL499606 & Winged Helix Forkhead & 1.3 & 25.2 \\
\hline 8 & FOXF2 & RP11-13/16 & Forkhead Box Protein F2 & & 25.2 \\
\hline 9 & FOXC1 & AL034344 & Forkhead Domain Transcription Factor & 1.56 & 25.2 \\
\hline 10 & GMDS & AL033517 & GDP Mannose,4,6 Dehydratase & 1.71 & 25.2 \\
\hline 11 & NOVEL & & Unknown & 1.89 & 25.2 \\
\hline Patient 5 & Distal & Breakpoint & & & \\
\hline & AL035693 & RP1-33B19 & & 2.07 & 25.2 \\
\hline 13 & 000366 & RP1-136B1 & Belongs to LINE1 & 2.4 & 25.2 \\
\hline 14 & NOVEL & RP1-136B1 & Reverse Transcriptase & 2.4 & 25.2 \\
\hline 15 & Q96MT4 & RP11-145H9 & Unknown & 2.6 & 25.2 \\
\hline 16 & NOVEL & RP11-145H9 & Myosin Light Chain Kinase,Smooth Muscle & & 25.2 \\
\hline 17 & Q8WV26 & AL139092 & A Werner Helicase Interacting Protein & 2.7 & 25.2 \\
\hline 18 & SERPIN B1 & RP11420G6 & $\begin{array}{l}\text { isoform } 1 \text {, } \\
\text { Monocyte/Neutrophil Elastase Inhibitor }\end{array}$ & 2.8 & 25.2 \\
\hline 19 & SERPIN B9 & AL133351(RP1-90|20) & $\begin{array}{l}\text { Cytoplasmic Antiproteinase } 3 \\
\text { Contur }\end{array}$ & 2.8 & 25.2 \\
\hline 20 & SERPIN BO & RP1-90)20 & Placental Thrombin Inhibitor & 2.9 & 25.2 \\
\hline 21 & Q96NM8 & RP1-90J20 & Unknown & 2.97 & 25.2 \\
\hline 22 & NQ02 & RP1-90J20 & Quinone Reductase 2(Dehydrogenase) & 3 & 25.2 \\
\hline 23 & NOVEL & RP1-40E16 & Unknown & 3 & 25.2 \\
\hline 24 & RIPK1 & RP1-40E16(D6S1338) & Receptor-interacting Serine/Threonine & 3.06 & 25.2 \\
\hline & & RP1-40F16(D6S1890) & Protein Kinase 2 & & 252 \\
\hline 26 & 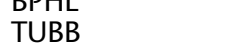 & $\begin{array}{l}\text { RPI-40E16(D6S1890) } \\
\text { RP1-40E16 }\end{array}$ & $\begin{array}{l}\text { Tubulin } \\
\text { Tulase-ike }\end{array}$ & $\begin{array}{l}3.1 \\
3.13\end{array}$ & 25.2 \\
\hline 27 & NOVEL & & Tubulin protein Family & 3.14 & 25.2 \\
\hline Patient 6 & Distal & Breakpoint & & & \\
\hline 28 & NOVEL & & Ion Transporter & 3.25 & 25.2 \\
\hline 29 & Q9UFY2 & RP11-15N12 & Ion Transporter Family & 3.3 & 25.2 \\
\hline 30 & NOVEL & RP1-72E17(D6S1196E) & Unknown & 3.7 & 25.2 \\
\hline 31 & Q9Y247 & & Belongs to XAP5 Protein HXC26 family & 3.8 & 25.2 \\
\hline 32 & Q9HD87 & AL1 38831 & Unknown & 3.96 & 25.2 \\
\hline 33 & Q8TDP2,Q9NQH: & RP5-1013A10(D6S1932) & Serine/Threonine protein kinase & & 25.2 \\
\hline 34 & NOVEL & D6S1142E & Unknown & 4.05 & 25.2 \\
\hline 35 & $\mathrm{PECl}$ & D6S1142E & Peroxisomal 3,2-trans-enoyl-coa isomerase & & 25.2 \\
\hline Patient 1 & Breakpoint & & & & \\
\hline & NOVEL & & Unknown & 4.1 & \\
\hline 37 & CDYL & RP5-1013A10 & Chromodomain protein, Y-chromosome like & 4.7 & 25.1 \\
\hline 40 & Q9BW22 & & Overlaps with CDYL & & 25.1 \\
\hline 41 & Q9U173 & RP3-430A16 & Unknown & 4.9 & 25.1 \\
\hline 42 & NOVEL & RP11-428J1 & & 4.9 & 25.1 \\
\hline 43 & NOVEL & AL359643 & Unknown & & 25.1 \\
\hline 44 & RP40 & RP11-428J1 & $\mathrm{A}$ ribonuclease $\mathrm{P}$ protein subunit $\mathrm{P} 40$ & 4.98 & 25.1 \\
\hline 45 & Q8WY88 & D6S1789 & Unknown & & 25.1 \\
\hline
\end{tabular}




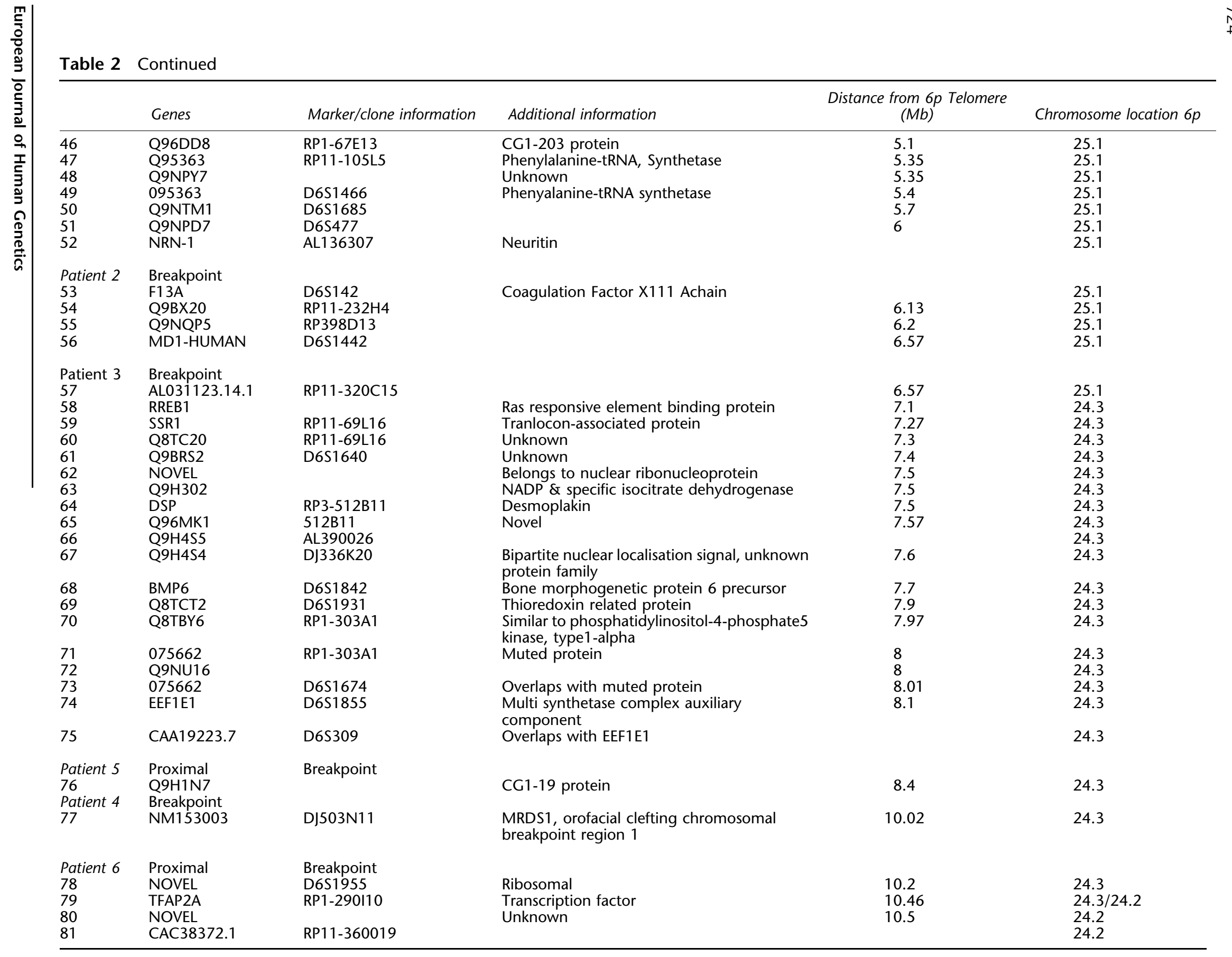


Table 3 List of probes used for in situ hybridisation on chromosomes of the $6 \mathrm{p}$ deletion cases

\begin{tabular}{|c|c|c|c|c|c|c|c|c|}
\hline Location on $6 p$ & Clones $6 p \mathrm{Tel}$ & Markers \& details & Patient 1 & Patient 2 & Patient 3 & Patient 4 & Patient 5 & Patient 6 \\
\hline 25.3 & $6 \mathrm{k} 23$ & & - & & - & & & \\
\hline 25.3 & DJ15h23 & (D6S1600)B76465GSS & & - & - & & + & + \\
\hline 25 & D)116 B8 & (D6S344)ÁL589989 & & & & & & + \\
\hline 25 & DJ118 B18\} & (870D6)Á034344(FOXC1) & - & - & & - & + & + \\
\hline 25 & $905 \mathrm{~F} 3$ & AFMBO34YA5 & - & & & & & \\
\hline 25 & 870D6 & AFMBO34YA5 & - & & & & & + \\
\hline 25 & DJ33 B19 & GMDS & & - & - & & + & \\
\hline 25 & Dj90 J20 & AL133351 & & - & - & & & \\
\hline 25 & D) 40 E16 & P1 REGION AL031963 & - & - & - & & & \\
\hline 25 & B20 & P19 & - & - & - & & - & + \\
\hline 25 & D)283 K9 & (AL1603980) & - & & - & & - & + \\
\hline 25 & D|262 P19 $\}$ & & - & - & - & & - & \\
\hline 25 & DJ223 B1 & AL0319433 & - & & & - & & - \\
\hline 25 & Dl72 E17 & AL38881 & - & & & & - & - \\
\hline 25 & $952 \mathrm{H} 4$ & AFMA339YD9 & + & & & & & \\
\hline 25 & D)52 F12 & AQ081478GSS & & & & & & \\
\hline 25 & D)21 P18 & AL1333939 & + & - & & & & - \\
\hline 25 & DJ36 I2 & PROX TO CDYL & + & - & & & - & - \\
\hline 25 & D)67 E13 & AL035653 & + & - & & - & & \\
\hline 25 & D)232 P20 & D6S1466 & & - & - & & & \\
\hline 25 & Dj182016 & D6S1685 & & - & - & & & \\
\hline 25 & D) $103 \mathrm{H} 18$ & D6S1677 & + & & & & & \\
\hline 25 & D) 287 K15 & D6S1574 \&(D6S477)(F13A) & & $-/+$ & - & - & & - \\
\hline 25 & 878 B 10 & D6S1574 & + & + & - & & & \\
\hline 25 & 663A12 & F13A & + & & - & & & \\
\hline 25 & $853 C 3$ & D6S1574 & + & + & & & & \\
\hline 25 & D)23 O21 & D6S1677 & + & + & $-/+$ & & & \\
\hline 25 & Dj80 N2 & PRP1 & & + & $-/+$ & - & - & \\
\hline 25 & 938B10 & D6S1598 & + & & & & & \\
\hline $25 / 24.3$ & D)129 N15 $\}$ & BMP 6 & & & + & - & - & - \\
\hline $25 / 24.3$ & Dj155 I9 & D6S1640 & & & + & - & & - \\
\hline $25 / 24.3$ & D)29 L9 & DSP & + & + & + & & - & - \\
\hline 24.3 & DJ164F5 & Wl-672 & & & + & & & - \\
\hline 24.3 & D) $133 \mathrm{H} 11$ & D6S410 & & & + & & - & \\
\hline 24.3 & DJ167K8 & STS34084 & & + & & & + & - \\
\hline 24.3 & Dj110P13 & SGC33794 & & & + & - & & - \\
\hline 24.3 & DJ135K15 & SGC33794 & & & & & & \\
\hline 24.3 & Dj103M22 & AL031904 & & & & & + & \\
\hline 24 & Dj90 012 & AL031906 & & + & & - & & \\
\hline 24.3 & Dj62A14 & STSG11171 & & + & + & - & + & - \\
\hline 24 & Dj436h5 & STSG 11216 & & + & & - & & \\
\hline 24 & ba648N19 & AL159986 & & + & + & + & & - \\
\hline 24 & DJ398a12 & AL021332 & & & & + & & \\
\hline 24 & D)359L13 & AL1359316 & + & & & + & & + \\
\hline 24 & Dj524G21 & STSG24768 & & + & + & + & & + \\
\hline 24 & DJ380L24 & AL139332 & & & & + & & + \\
\hline $24.1-24.3$ & Dj503N11 & AL031122 & & & & & & + \\
\hline 24 & D) $242 F 16$ & STSG1197 & & & & & & + \\
\hline $24.1-24.3$ & DJ93h4 & AL031122 & & & & & & + \\
\hline 24 & D) $151 \mathrm{c} 13$ & STSG26237 & & & & + & & + \\
\hline 24 & Dj172h1 & STS23597 & & & & & + & + \\
\hline 24 & Dj290I10 & TFAP2a & & & & + & & + \\
\hline 24.2 & 938D8 & AP2,D6S470 & & & & & & + \\
\hline 24.1 & $886 \mathrm{~A} 2$ & D6S429 & + & & & & & \\
\hline 23 & $772 \mathrm{~B} 2$ & D6S289 & & & & + & & + \\
\hline 23 & $912 \mathrm{G} 9$ & D6S1667 & & & & & & + \\
\hline 23 & $683 \mathrm{H} 6$ & CD83,D6S259 & & + & & & & + \\
\hline 23 & 930D2 & JMJ,AFMA139WE5 & & & & + & & + \\
\hline 23 & D)149A2 & SCA1 & + & & & & + & \\
\hline
\end{tabular}

The cytogenetic position of the probes is indicated (left column), the name of the clones and information about marker content (middle column) and hybridisation results on patient's derivative 6 chromosomes (columns 1-6 to the right). Hybridisation (+) or failure of hybridisation ( - ) of probes to the derivative chromosome 6 is shown. A weak signal on the derivative 6 is described as $+/-$, indicating the breakpoint is close. The clones have been positioned in order using FISH and according to the Sanger centre database (http://www.sanger.ac.uk/cgi-bin/humace/fpcwebmap). 

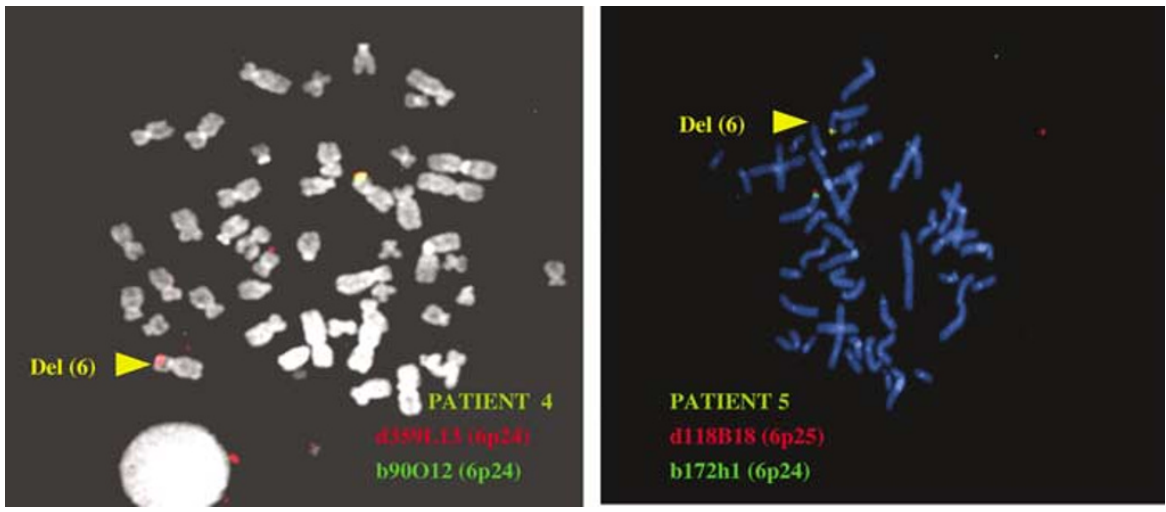

Figure 2 Examples of results showing metaphase chromosomes prepared from a selection of cases under investigation. Probes used for in situ hybridisation were labelled with biotin or digoxygenin and detected using fluorescein isothiocyanate or tetramethylrhodamine isothiocyanate systems. Hybridisation signals are indicated by arrowheads. (Left) Mapping the terminal deletion of Patient 4; PAC 359L13 (mapping in 6p24, red) hybridised to both chromosome 6 and del (6), whereas the more distal Pac 90012 (also mapping to 6p24, green) hybridised only to the normal chromosome 6 . Therefore the breakpoint lies between these two clones within band 6p24. (Right) Mapping the interstitial deletion in Patient 5; PAC118B18(red), mapping in $6 \mathrm{p} 25$, hybridised to both chromosome 6 and del (6), and PAC 172H1 (green) hybridised proximal to PAC118B18 within $6 \mathrm{p} 24$ on normal chromosome $6 \mathrm{p}$. A green signal derived from PAC $172 \mathrm{H} 1$ is also present on del (6), and overlaps with the red signal (PAC118B18) in 6p25, indicating that a significant proportion of the region distal to $6 \mathrm{p} 24$ and proximal to $6 \mathrm{p} 25$ has been deleted.

\section{Discussion}

A number of genes have now been localised to the short arm of human chromosome 6 , and current available data for this region have been analysed in an effort to gain direct relevance to our cases (Table 2). In addition, progress has been made in creating knockout mice for specific genes ${ }^{12,17}$ or mice carrying defined chromosomal deletions. ${ }^{14}$ Phenotypic characterisation of these mice and comparison to human patients with relevant chromosomal deletions enables us to extract information about gene function in this region. The main phenotypes observed in the cases described here are discussed below:

\section{Ocular defects}

Our four $6 \mathrm{p}$ terminal deletion cases exhibit ocular abnormalities, sensorineuronal hearing loss and developmental delay (clinical findings that constitute the description of $6 \mathrm{p}$ terminal deletion syndrome). ${ }^{1}$ Although the extent of the deletion is different in each case, all are haploinsufficient for the region on $6 \mathrm{p} 25$ containing FOXC1 and associated with disorders of the eye, namely iridogoniodysgenesis, Axenfeld-Rieger anomaly/syndrome, primary congenital glaucoma and iris hypoplasia. ${ }^{18,19}$ The variable penetrance of the eye anomalies observed in our $6 \mathrm{p}$ terminal deletion cases may be explained by different genetic backgrounds in agreement with the mouse model, ${ }^{14}$ but parental origin effects, stochastic developmental events or mutations in other genes of related function may contribute. In addition, the ocular finding of grey-blue sclerae (Patients 5 and 6) can be associated with deletion of the 6p24-p25 segment distal to AP-2 and proximal to FOXC1 (Tables 1 and 2).

\section{Hydrocephalus}

The mouse Mf1 (FOXC1) knockout model and the allelic ch mouse show pleiotropic skeletal and ocular phenotypes. $^{12,14}$ These mice on various genetic backgrounds present craniofacial defects, lethal congenital hydrocephalus, eye defects and abnormalities of the central nervous, urogenital and skeletal systems. Parts of these phenotypes, like the ocular defects, can occur in mice heterozygous for the Foxc1 mutation. ${ }^{15}$ Patient 4 presents with congenital hydrocephalus, heart and anterior eye developmental defects, showing considerable similarity to the congenital hydrocephalus (ch) mouse model. It would appear that by comparing our four terminal deletion cases (Table 3), hemizygosity for both Bone morphogenetic protein 6 (BMP6) and the FOXC1 gene produces such a phenotype as hypothesised by Kume et al. ${ }^{12}$ However, it is worth noting that the case reported by Navarro et $a l^{3}$ (and GK Mirza, AM Perle and J Ragoussis, unpublished results) and an unbalanced translocation case (CA in Davies et $a l^{1}$ ), present with hydrocephalus associated with the deletion of FOXC1, while BMP 6 is retained. Therefore, we can conclude that the factors determining the occurrence of hydrocephalus could be more complex than the contiguous deletion of the FOXC1-BMP6 chromosomal segment. 


\section{Heart defects}

Patient 1 and one of our published $6 \mathrm{p}$ terminal deletion cases, ${ }^{1}$ carry deletions of FOXC1 and present with aortic valve abnormalities. Furthermore, aortic valve abnormalities have been diagnosed in the FOXC1 mouse model. ${ }^{13}$ Thus we conclude that FOXC1 hemizygosity appears to be an important determinant of aortic valve development. Suggestions that evolve from analysis of cases in the literature with molecular characterisations are that regions deleted proximal of $6 \mathrm{p} 25$ attribute to other heart defects, in particular ASD, VSD and PDA. In combination with Patients 5 and 6 , we suggest the involvement of other loci positioned between $6 \mathrm{p} 24.3$ and $6 \mathrm{p} 25$ in heart development. Of the genes identified within the relevant chromosomal segment, BMP6 is a possible candidate since it is expressed in the heart. ${ }^{17}$

\section{Bone formation}

(a) Joint formation Three cases presented with joint hyperlaxity; Patients 2, 5 and 3. The latter case presented with osteochondritis juvenilis deformans (Perthes disease). Additional reports, including the case described by Guillen-Navarro ${ }^{3}$ and an unbalanced translocation case (BD in Davies et $a l^{1}$ ), all with deletion of $6 \mathrm{p} 25$, present with delayed bone maturation and hip dislocations. In the case of Patients 2 and 3, these findings agree with the Mf1 knockout model; however, Patient 5 presents evidence for other genes further centromeric that contribute to chondrogenesis.

(b) Pectus excavatum A further interesting aspect of Patients 5 and 6 is that the segment containing the BMP6 gene is deleted. Both cases exhibit pectus excavatum, consistent with the phenotypic finding in one of our previously reported cases carrying a deletion of BMP6. ${ }^{9}$ Mice lacking BMP6 (the heterozygotes are reported normal) show defects in the development of the sternum ${ }^{17}$ indicating that the human protein may have a similar function to the mouse homologue.

Hearing defects Our $6 \mathrm{p}$ terminal deletion cases 1,3 and 4 present with hearing loss, and case 2 demonstrates auditory hypersensitivity. The interstitial deletion cases lack such symptoms and therefore we suggest that a gene involved in hearing development can be positioned distal to the most telomeric interstitial breakpoint observed (case 5 ), that is, distal to the region containing the GMDS gene (Tables 2 and 3).

Craniofacial anomalies Our interstitial deletion cases, 5 and 6 , are reported present with a highly arched palate and a cleft lip, respectively: the proximal breakpoints of both deletions lie in $6 \mathrm{p} 24.3$. A number of studies have shown linkage to this region for orofacial clefting. ${ }^{20-22}$ Patient 2 is the first case to have both cleft lip and palate and this could result from an interaction between loci in $6 \mathrm{p} 24$ and $6 \mathrm{p} 25$. In summary these cases support the evidence that genes contributing to orofacial clefting lie distal to AP2 and proximal to FOXC1 gene.

Skin defects Haploinsufficiency for the desmoplakin (DSP) gene has been implicated in causing an inherited skin disorder, palmoplanter keratoderma. Patients 4, 5 and 6 are hemizygous for this gene. Only the latter case showed defects to the skin, namely eczema. Other related cases have reported excessively dry skin but none so far have reported palmoplantar keratoderma. We conclude that hemizygosity for DSP on its own is not sufficient to cause this disease.

Cognitive/neuromotor delay With the exception of Patient 1, our cases are hemizygous for the gene neuritin (Table 2) and except for Patient 6, our cases present with either neuronal or motor development delay. Patient 2 presents with epilepsy. Neuritin is induced by neuronal activity and promotes neuritogenesis; ${ }^{23}$ therefore, haploinsufficiency for this gene may contribute to developmental delay in our cases.

Tooth development defects Our terminal deletion patients, 1, 2 and 3, all show tooth abnormalities; Patients 5 and 6 have normal dentition. We can conclude that hemizygosity for the region between the most telomeric breakpoint in the vicinity of marker D6S1617 and the telomeric breakpoint of patient 6 results in such defects.

An important point that requires highlighting is that all cases have deletions that arose de novo. Patient 2 and our previous cases $\mathrm{BD}, \mathrm{GD}, \mathrm{HH}$ and SG have deletion breakpoints within the F13A gene. ${ }^{1}$ This part of the chromosome contains the fra (6) 6p25.1 common, aphidicolin-type fragile site, and it is of interest to fine map and characterise this site and to determine whether it is involved in the deletions presented here. The extent of deletion in our cases does not correlate with the severity of the phenotype (Tables 2 and $3 b$ ). Patient 1 has the most severe phenotype, but the least amount of deleted genome compared to the other five cases. Genetic background differences, parent-oforigin effects or undetected mosaicism may be responsible for the lack of correlation between phenotype severity and size of the deletions in our cases.

It is clear that the generation and detailed study of mouse models and human deletion cases have contributed significantly to understanding the molecular basis of chromosome $6 \mathrm{p}$ deletion syndromes. Further work involving generation of mutations in genes located within the segment syntenic to human chromosome $6 \mathrm{p}$ in mice will increase our understanding of the function and nature of developmental defects associated with these deletions (http://www.mgc.har.mrc.ac.uk/physical/del36h.html). 
Further details and links on $6 \mathrm{p}$ deletion cases are available at our website (http://www.well.ox.ac.uk/genomics).

\section{Acknowledgements}

We are, as ever, extremely grateful to the families of the patients. We thank Drs J Luck and J Sidman for their diagnosis of the respective cases, and thank Dr Zameel Cader and Dr Harris Khawaja for their review of the manuscript. This work was supported by the MRC Grant No. G9533412 and by the Wellcome Trust Grant No. GR060759MA.

\section{References}

1 Davies AF, Mirza G, Sekhon G et al: Delineation of two distinct 6p deletion syndromes. Hum Genet 1999; 104: 64-72.

2 Kasai O: A case of partial monosomy 6p. Jpn J Hum Genet 1982; 27: 214-215.

3 Guillen-Navarro: A rare de novo microdeletion of distal chromosome 6p: clinical phenotype and molecular cytogenetic characterisation. Am J Hum Genet 1997; 61: A719.

4 Anderlid B-M, Schoumans J, Hallqvist A et al: Cryptic subtelomeric $6 \mathrm{p}$ deletion in a girl with congenital malformations and severe language impairment. Eur J Hum Genet 2003; 11: 89-92.

5 Batanian JR, Grange DK, Fleming $\mathrm{R}$ et al: Two unbalanced translocations involving a common $6 \mathrm{p} 25$ region in two $\mathrm{XY}$ female patients. Clin Genet 2001; 59: 52-57.

6 Sivak LE, Esbenshade J, Brothman AR et al: Multiple congenital anomalies in a man with $(\mathrm{X} ; 6)$ translocation. Am J Med Genet 1994; 51: 9-12.

7 Narahara K, Seno Y, Nishibayashi Y et al: Gene dosage effects for coagulation factors XII (F12) AND XIII and subunit A (F13A) in a case with partial monosomy $6 \mathrm{p}$ resulting from a maternal pericentric inversion of chromosome 6. Jpn J Hum Genet 1987; 32: $305-310$.

8 Davies AF, Olavesen MG, Stephens RJ et al: A detailed investigation of two cases exhibiting characteristics of the $6 \mathrm{p}$ deletion syndrome. Hum Genet 1996; 98: 454-459.

9 Davies AF, Mirza G, Flinter F et al: An interstitial deletion of 6p24-p25 proximal to the FKHL7 locus and including AP-2alpha that affects anterior eye chamber development. $J$ Med Genet 1999; 36: 708-710.
10 Moriarty AP, Kerr-Muir MG: Sclerocornea and interstitial deletion of the short arm of chromosome 6- -(46XY del [6] [p22 p24]). J Pediatr Ophthalmol Strabismus 1992; 29: 177-179.13.

11 Topping A, Harris P, Moss AL: The 6p deletion syndrome: a new orofacial clefting syndrome and its implications for antenatal screening. Br J Plastic Surg 2002; 55: 68-72.

12 Kume T, Deng KY, Winfrey V et al: The forkhead/winged helix gene Mf1 is disrupted in the pleiotropic mouse mutation congenital hydrocephalus. Cell 1998; 93: 985-996.

13 Winnier GE, Kume T, Deng K et al: Roles for the winged helix transcription factors MF1 and MFH1 in cardiovascular development revealed by nonallelic noncomplementation of null alleles. Dev Biol 1999; 213: 418-431.

14 Arkell RM, Cadman M, Marsland T et al: Genetic, physical, and phenotypic characterization of the Del(13)Svea36H mouse. Mamm Genome 2001; 12: 687-694.

15 Hong HK, Lass JH, Chakravarti A: Pleiotropic skeletal and ocular phenotypes of the mouse mutation congenital hydrocephalus (ch/Mf1) arise from a winged helix/forkhead transcriptionfactor gene. Hum Mol Genet 1999; 8: 625-637.

16 Hong HK, Noveroske JK, Headon DJ et al: The winged helix/ forkhead transcription factor Foxq1 regulates differentiation of hair in satin mice. Genesis 2001; 29: 163-171.

17 Solloway MJ, Dudley AT, Bikoff EK et al: Mice lacking Bmp6 function. Dev Genet 1998; 22: 321-339.

18 Nishimura DY, Swiderski RE, Alward WL et al: The forkhead transcription factor gene FKHL7 is responsible for glaucoma phenotypes which map to 6p25. Nat Genet 1998; 19: 140-147.

19 Mirzayans F, Gould DB, Heon E et al: Axenfeld-Rieger syndrome resulting from mutation of the FKHL7 gene on chromosome 6p25. Eur J Hum Genet 2000; 8: 71-74.18.

20 Scapoli L, Pezzetti F, Carinci F et al: Evidence of linkage to $6 \mathrm{p} 23$ and genetic heterogeneity in nonsyndromic cleft lip with or without cleft palate. Genomics 1997; 43: 216-220.

21 Prescott NJ, Lees MM, Winter RM et al: Identification of susceptibility loci for nonsyndromic cleft lip with or without cleft palate in a two stage genome scan of affected sib- pairs. Hum Genet 2000; 106: 345-350.

22 Davies AF, Stephens RJ, Olavesen MG et al: Evidence of a locus for orofacial clefting on human chromosome 6p24 and STS content map of the region. Hum Mol Genet 1995; 4: 121-128.

23 Naeve GT, Ramakrishnan M, Kramer R et al: Neuritin: A gene induced by neural activity and neurotrophins that promotes neuritogenesis. Proc Natl Acad Sci USA 1997; 94: 2648-2653. 